\title{
Nucleon-to-resonance transition form factors in a vector-meson-dominance model
}

\section{Grigoriy Vereshkov and Nikolay Volchanskiy*}

Research Institute of Physics, Southern Federal University, Rostov-na-Donu, 344090, Russia

E-mail: nikolay.volchanskiy@gmail.com, gveresh@gmail.com

\begin{abstract}
We study the $Q^{2}$-evolution of the form factors (FFs) for the nucleon-to-resonance transitions in the framework of an effective field theory. To this end, the intrinsic symmetries of the RaritaSchwinger (RS) fields are analyzed, and a Lagrangian of the electromagnetic $N R$-interactions is constructed. The Lagrangian preserves all the intrinsic symmetries of the higher-spin RS fieldpoint and gauge invariance-and does not involve lower-spin components of the reducible RS field. Besides, the symmetries postulate the definitions of the Lagrangian FFs. These FFs are modeled as dispersionlike expansions in a vector-meson-dominance model. A good agreement with the experimental data is achieved.
\end{abstract}

The XXth International Workshop High Energy Physics and Quantum Field Theory September 24 - October 1, 2011

Sochi, Russia

\footnotetext{
* Speaker.
} 


\section{Introduction}

To investigate the $Q^{2}$-dependence of the nucleon-to-resonance transition in the framework of the effective field theory, we need to specify an effective baryon-meson vertex for the radiative $N R$-transitions. The vertex determines relations between experimentally measurable helicity amplitudes $\mathscr{A}\left(Q^{2}\right), \mathscr{A}=A_{3 / 2}, A_{1 / 2}, S_{1 / 2}$ and a set of the transition FFs $F_{f}\left(Q^{2}\right), f=1,2,3$ that are free of kinematic factors,

$$
\mathscr{A}\left(Q^{2}\right)=\mathscr{A}\left[Q^{2}, F_{1}\left(Q^{2}\right), F_{2}\left(Q^{2}\right), F_{3}\left(Q^{2}\right)\right]
$$

The relations (1.1) comprise two types of $Q^{2}$-dependent functions. The functions of the first type are specified by defining the effective vertex of the $N R$-interactions with photons and vector mesons. Thus, the physical meaning of the FFs can depend upon the definition of the vertex, since different relations (1.1), if resolved in respect to $F_{f}\left(Q^{2}\right)$, may result in different $Q^{2}$-behavior of the FFs. We address constructing the vertexes in Sec. 2. The second type of functions are the transition FFs $F_{f}\left(Q^{2}\right)$ to be evaluated in QCD or phenomenological approaches. In Sec. 3 we consider the FFs in a vector-meson-dominance model.

\section{Lagrangians of the higher-spin-resonance electroproduction}

In the effective field theory higher spin baryon resonances are commonly described in terms of the Rarita-Schwinger (RS) fields. These fields possess some specific symmetry properties [1]. In the massless limit the Lagrangian of the free RS field of the tensor rank $\ell$ is invariant under the gauge transformations $\Psi \rightarrow \Psi+\operatorname{sym} \partial \theta$, where $\theta$ is a field of the rank $\ell-1$. Besides, the free RS field equations are produced by an equivalent class of the Lagrangians that is invariant under a nonunitary symmetry group of the "point" transformations $\Psi \rightarrow \Psi+\operatorname{sym} \gamma \theta$, where $\gamma$ are the Dirac matrices. The symmetries are inextricably linked to the constraints imposed on the field to do away redundant degrees of freedom. However, in the presence of interactions, the symmetries of the free RS field can be easily broken. It is well known that such interactions break the symmetries, modify covariant constraints, and result in different pathologies including superluminal propagation and problems with quantization [2-5].

It is possible to prevent the aforementioned inconsistencies, if we require the invariance of the interaction Lagrangian under the point and gauge transformations of the RS field $\Psi \rightarrow \Psi+$ sym $\partial \theta_{1}+\operatorname{sym} \gamma \theta_{2}$, where $\theta_{1}, \theta_{2}$ are fields of the rank $\ell-1$. One of the mathematical consequences of the symmetry is that both the RS field and tensor-spinor interaction current satisfy the conditions of transversality and (spinor) tracelessness:

$$
\partial^{\lambda} \Psi_{\lambda \mu_{2} \ldots \mu_{\ell}}=0=\gamma^{\lambda} \Psi_{\lambda \mu_{2} \ldots \mu_{\ell}}, \quad \partial^{\lambda} J_{\lambda \mu_{2} \ldots \mu_{\ell}}=0=\gamma^{\lambda} J_{\lambda \mu_{2} \ldots \mu_{\ell}}
$$

The point and gauge invariant interactions are free from the pathologies, because the conditions (2.1) eliminate all the redundant lower-spin degrees of freedom of the RS field. 
The minimally local point and gauge invariant Lagrangian of the electromagnetic nucleonresonance interactions can be written for the resonances of the spin $J=\ell+1 / 2$ as follows

$$
\begin{gathered}
\mathscr{L}=\mathscr{L}_{1}+\mathscr{L}_{2}+\mathscr{L}_{3}+\text { h.c. } \\
\mathscr{L}_{1}=\frac{i^{3 \ell+2} g_{1}}{2 M_{N}^{3 \ell-1} \bar{\Psi}^{\bar{A}, \lambda_{2} \ldots \lambda_{\ell}} \Gamma_{\bar{A} \bar{B}}^{(\ell)} \gamma_{R} N^{,} \sigma_{2} \ldots \sigma_{\ell} V^{\lambda_{1} \sigma_{1}},} \\
\mathscr{L}_{2}=\frac{i^{3 \ell+3} g_{2}}{2 M_{N}^{3 \ell-1} M_{R}} \bar{\Psi}^{\bar{A}, \lambda_{2} \ldots \lambda_{\ell} \rho} \Gamma_{\bar{A} \bar{B}}^{(\ell)} \gamma_{\rho} \gamma_{R} N^{, \sigma_{2} \ldots \sigma_{\ell}} V^{\lambda_{1} \sigma_{1}}, \\
\mathscr{L}_{3}=\frac{i^{3 \ell+2} g_{3}}{2 M_{N}^{3 \ell-1} M_{R}^{2}} \bar{\Psi}^{\bar{A}, \lambda_{2} \ldots \lambda_{\ell} \rho}\left(\Gamma_{\bar{A} \bar{B}^{1} \lambda_{1} \rho}^{(\ell)} g_{\sigma_{1} \omega}-\Gamma_{\bar{A} \bar{B}^{1} \sigma_{1} \rho}^{(\ell)} g_{\lambda_{1} \omega}+\Gamma_{\bar{A} \bar{B}^{1}\left[\lambda_{1} \omega\right]}^{(\ell)} g_{\sigma_{1} \rho}-\Gamma_{\bar{A} \bar{B}^{1}\left[\sigma_{1} \omega\right]}^{(\ell)} g_{\lambda_{1} \rho}\right. \\
\\
\left.-\Gamma_{\bar{A} \bar{B}^{1}\left[\lambda_{1} \sigma\right]}^{(\ell)} g_{\rho \omega_{1}}\right) N^{, \sigma_{2} \ldots \sigma_{\ell} \omega} V^{\lambda_{1} \sigma_{1}} .
\end{gathered}
$$

Here $\bar{A}=\left(\left[\mu_{1} v_{1}\right]\left[\mu_{2} v_{2}\right] \ldots\left[\mu_{\ell} v_{\ell}\right]\right), \bar{B}=\left(\left[\lambda_{1} \sigma_{1}\right]\left[\lambda_{2} \sigma_{2}\right] \ldots\left[\lambda_{\ell} \sigma_{\ell}\right]\right)$, and $\bar{B}^{a}=\left(\left[\lambda_{1} \sigma_{1}\right] \ldots\left[\lambda_{a-1} \sigma_{a-1}\right]\right.$ $\left.\left[\lambda_{a+1} \sigma_{a+1}\right] \ldots\left[\lambda_{\ell} \sigma_{\ell}\right]\right)$ are multi-indices; $\Psi^{\bar{A}}$ is a gauge invariant tensor spinor made of the RS field operator and $\ell$ derivatives; $V^{\lambda_{1} \sigma_{1}}$ are field tensors of the vector fields $V=\gamma, \rho(770), \omega(785)$, $\rho(1450), \ldots ; \gamma_{R}=1$ for tensor-spinor resonances $J^{P}=(3 / 2)^{-},(5 / 2)^{+}, \ldots$ and $\gamma_{R}=i \gamma_{5}$ for pseudotensor-spinor ones $J^{P}=(3 / 2)^{+},(5 / 2)^{-}, \ldots$ For the lowest spins $J=3 / 2,5 / 2$ the Lagrangian (2.2) was constructed in Refs. [6, 7].

All three terms of the Lagrangian (2.2) comprise the unique vertex matrix $\Gamma_{\bar{A} \bar{B}}^{(\ell)}$ that is $\gamma$ transversal due to the symmetry of the Lagrangian: $\gamma^{\mu_{1}} \Gamma_{\left(\left[\mu_{1} v_{1}\right] \ldots\left[\mu_{\ell} v_{\ell}\right]\right)\left(\left[\lambda_{1} \sigma_{1}\right] \ldots\left[\lambda_{\ell} \sigma_{\ell}\right]\right)}^{(\ell)}=0$. In the simplest case of the vector spinor resonances $J=3 / 2(\ell=1)$ the vertex matrix is

$$
\Gamma_{[\mu v][\lambda \sigma]}=-\frac{1}{6}\left(\sigma_{\mu v} \sigma_{\lambda \sigma}+3 \sigma_{\lambda \sigma} \sigma_{\mu v}\right) .
$$

For higher ranks $\ell$ the vertex matrices are defined by a recurrence relation:

$$
\Gamma_{\bar{A} \bar{B}}^{(\ell)}=\frac{3}{2(2 \ell+1) \ell^{2}} \sum_{a, b=1}^{\ell}\left[(\ell+1) \Gamma_{\bar{A}^{a} \bar{B}^{b}}^{(\ell-1)} \Gamma_{A^{a} B^{b}}^{(1)}+(\ell-1) \Gamma_{\bar{A}^{a} \bar{B}^{b}}^{(\ell-1)} \Gamma_{B^{b} A^{a}}^{(1)}+\sum_{b \neq c=1}^{\ell} \Gamma_{\bar{A}^{a} A^{a} \bar{B}^{b c}}^{(\ell-1)} \Gamma_{B^{b} B^{c}}^{(1)}\right],
$$

where $A^{a}=\left[\mu_{a} v_{a}\right]$ and $B^{a}=\left[\lambda_{a} \sigma_{a}\right]$. Therefore, in the point and gauge invariant theory the tensor spinor $\Gamma_{[\mu v][\lambda \sigma]}^{(1)}$ plays a special role similar to that of the Dirac matrices $\gamma_{\mu}$ in the theory of the spin- $1 / 2$ interactions - all the $\gamma$-transversal vertex matrices are expanded in terms of the simplest matrix $\Gamma_{[\mu v][\lambda \sigma]}^{(1)}$.

The helicity amplitudes for the mass-shell electroproduction of the resonance of the spin $J=$ $\ell+1 / 2$ calculated using the point and gauge invariant Lagrangian (2.2) are

$$
\begin{aligned}
& A_{3 / 2}^{(p, n)}\left(Q^{2}\right)=\mp \sqrt{N_{\ell}}\left[\left(Q^{2} \pm \mu_{ \pm} M_{N}\right) F_{1}^{(p, n)}\left(Q^{2}\right)+\mu_{ \pm} M_{R} F_{2}^{(p, n)}\left(Q^{2}\right)-\left(Q^{2}+\mu_{ \pm} M_{R}\right) F_{3}^{(p, n)}\left(Q^{2}\right)\right], \\
& A_{1 / 2}^{(p, n)}\left(Q^{2}\right)=-\sqrt{\frac{\ell N_{\ell}}{\ell+2}}\left[\mu_{ \pm} M_{R} F_{1}^{(p, n)}\left(Q^{2}\right)+\left(Q^{2} \pm \mu_{ \pm} M_{N}\right) F_{2}^{(p, n)}\left(Q^{2}\right) \mp \mu_{ \pm} M_{N} F_{3}^{(p, n)}\left(Q^{2}\right)\right], \\
& S_{1 / 2}^{(p, n)}\left(Q^{2}\right)=\mp \sqrt{\frac{\ell N_{\ell}}{2(\ell+2)}} Q_{+} Q_{-}\left[F_{1}^{(p, n)}\left(Q^{2}\right)-F_{2}^{(p, n)}\left(Q^{2}\right)+\frac{Q^{2}+M_{R}^{2}+M_{N}^{2}}{2 M_{R}^{2}} F_{3}^{(p, n)}\left(Q^{2}\right)\right],
\end{aligned}
$$


where $N_{\ell}=\frac{2^{\ell}(\ell !)^{2} \pi \alpha Q_{ \pm}^{2(\ell-1)} Q_{\mp}^{2 \ell}}{(2 \ell) ! M_{N}^{4+1}\left(M_{R}^{2}-M_{N}^{2}\right)}, \mu_{ \pm}=M_{R} \pm M_{N}$, and $Q_{ \pm}=\sqrt{Q^{2}+\mu_{ \pm}^{2}}$. In the above equation the upper and bottom signs correspond to the resonances with the spin-parities $J^{P}=(3 / 2)^{ \pm},(5 / 2)^{\mp}, \ldots$, respectively.

The perturbative QCD scaling predictions [8-10] for the helicity amplitudes (2.5) result in the following constraints on the FFs at high $Q^{2}$

$$
F_{1}\left(Q^{2}\right) \sim \frac{1}{Q^{6+2 \ell} L^{n_{1}}\left(Q^{2}\right)}, \quad F_{2}\left(Q^{2}\right) \sim \frac{1}{Q^{4+2 \ell} L^{n_{2}}\left(Q^{2}\right)}, \quad F_{3}\left(Q^{2}\right) \sim \frac{1}{Q^{6+2 \ell} L^{n_{3}}\left(Q^{2}\right)} .
$$

where $L^{n_{f}}\left(Q^{2}\right)=\ln ^{n_{f}}\left(Q^{2} / \Lambda^{2}\right)$ and $n_{2}-n_{3} \approx 2[10]$.

\section{FFs in a vector-meson-dominance model}

Within the vector-meson-dominance (VMD) model [11], the FFs $F_{f}\left(Q^{2}\right)$ are given by dispersionlike expansions

$$
F_{f}^{(p, n)}\left(Q^{2}\right)=\frac{1}{2}\left[\sum_{k=1}^{K} \frac{\varkappa_{k f}^{(\omega)}\left(Q^{2}\right) m_{(\omega) k}^{2}}{Q^{2}+m_{(\omega) k}^{2}} \pm \sum_{k=1}^{K} \frac{\varkappa_{k f}^{(\rho)}\left(Q^{2}\right) m_{(\rho) k}^{2}}{Q^{2}+m_{(\rho) k}^{2}}\right],
$$

with the poles being at the masses $m_{(\rho, \omega) k}$ of the experimentally observed vector mesons $\rho(770)$, $\omega(782), \rho(1465), \rho(1425) \ldots$ In Eq. (3.1) we introduce $Q^{2}$-dependencies of the baryon-meson parameters $\varkappa_{f}^{(\omega, \rho)}\left(Q^{2}\right)$, which phenomenologically takes into account the influence of the smallscale quark-gluon effects on the photon hadronization in the nucleon medium for $Q^{2}>R_{\text {nucl }}^{-2}=$ $(0.2 \mathrm{GeV})^{2}$.

To assure correct high- $Q^{2}$ behavior (2.6) of the dispersionlike expansions of the FFs (3.1), we assume the following: (i) The $Q^{2}$-dependence of the expansion coefficients is independent of the meson-family index $k, \varkappa_{k f}\left(Q^{2}\right)=\varkappa_{k f}(0) / L_{f}\left(Q^{2}\right)$. (ii) The interpolation functions $L_{f}\left(Q^{2}\right)$ are given by $L_{f}=\left(1+b_{f} \bar{\ell}+a_{f} \bar{\ell}^{2}\right)^{n_{f} / 2}, \bar{\ell}=\ln \left(1+Q^{2} / \Lambda^{2}\right)$, which effectively takes into account the renormalization of the strong coupling constant. (iii) The parameters of the meson spectrum satisfy the superconvergence relations $\sum_{k=1}^{K} m_{(\omega, \rho) k}^{2 n} \varkappa_{k f}^{(\omega, \rho)}(0)=0$, where $n=2,3, \ldots 4+\ell$ for $f=1,3$ and $n=2, \ldots 3+\ell$ for $f=2$ in the case of electroproduction of a resonance with the spin $J=\ell+\frac{1}{2}$.

The dispersionlike expansions of the FFs are fitted to the experimental data on the electroproduction of the resonances $\Delta(1232), N^{*}(1440), N^{*}(1520), N^{*}(1535), N^{*}(1680)$ off the proton. In the fit we use $K=5$ vector meson families. This is the minimal number of the mesons that is sufficient to saturate the superconvergence relations. The fit curves and the data points are depicted in Figs. 1-5. The references to the experimental data have been collected in $[6,7,11]$.

\section{Conclusion}

We have constructed an effective-field model of the electromagnetic $N R$-transitions. The interaction Lagrangian of the model preserves all the symmetries of the free RS field and, consequently, does not modify the free-field constraints. The Lagrangian belongs to the class of consistent gauge-invariant interactions [5], which do not excite redundant lower-spin components of 


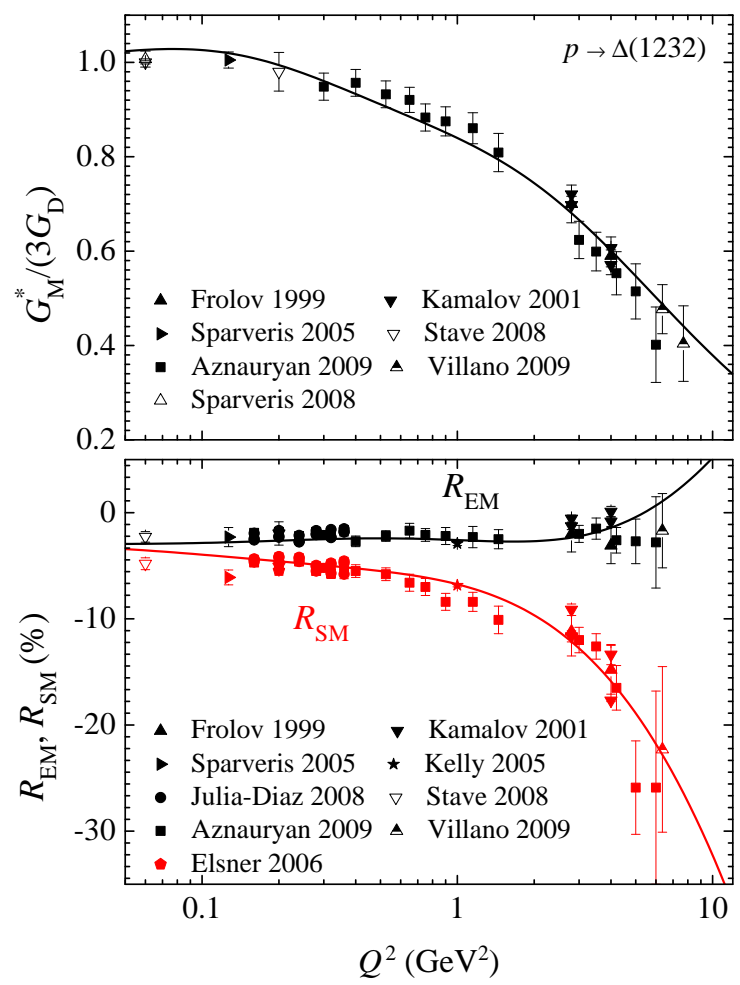

Figure 1: Magnetic FF and the amplitude ratios for the transition $p \gamma^{*} \rightarrow \Delta(1232)\left(\chi^{2} / D O F=1.51\right)$
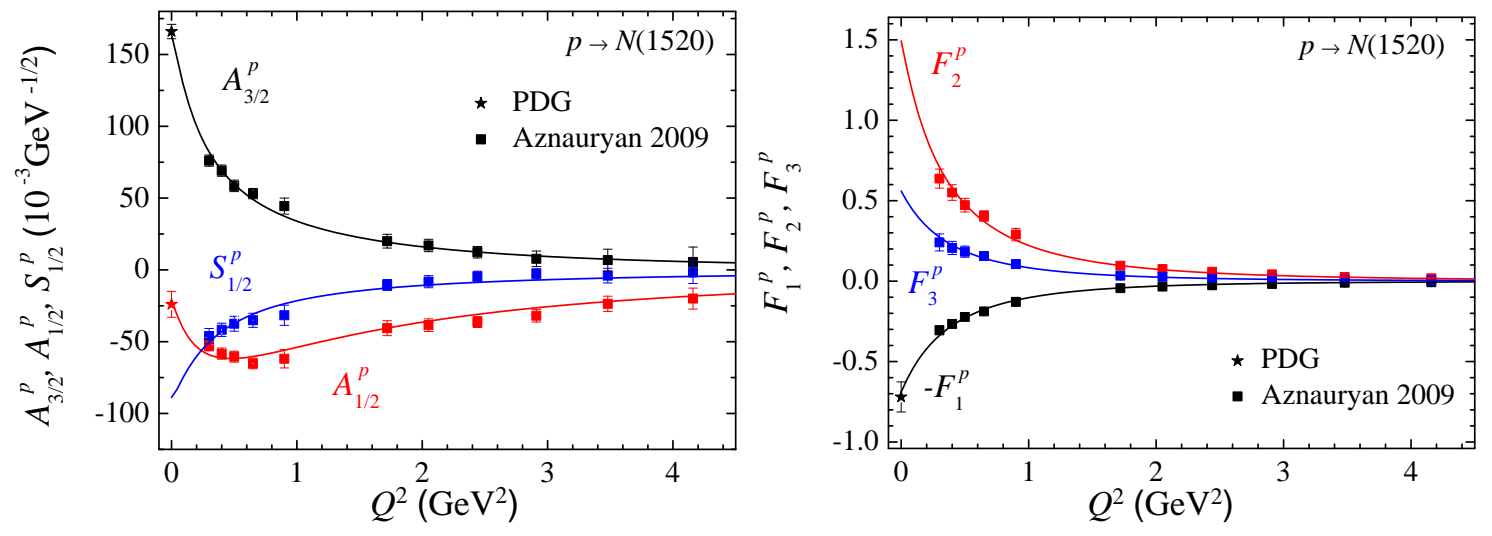

Figure 2: Helicity amplitudes and point and gauge invariant FFs for the transition $p \gamma^{*} \rightarrow N_{+}^{*}(1520)$ $\left(\chi^{2} / D O F=1.05\right)$

the RS field. Being point-invariant as it is, the Lagrangian is specific among other possible gaugeinvariant Lagrangians: (1) All three terms of the minimally local Lagrangian are defined uniquely by the symmetry and their differential order. (2) The symmetry classifies the FFs in terms of the differential oder of the corresponding Lagrangian vertex.

Besides, the point and gauge invariant $\gamma^{*} N R$-interactions are unified in three ways: (1) All tensor-spinor couplings are expressed through the universal tensor spinor (2.3). (2) The vertex matrix (2.4) takes the same place in the Lagrangians (2.2) for arbitrarily high resonance spin. (3) The pre-FF polynomials in the helicity amplitudes (2.5) do not depend on the spin of the resonance. 

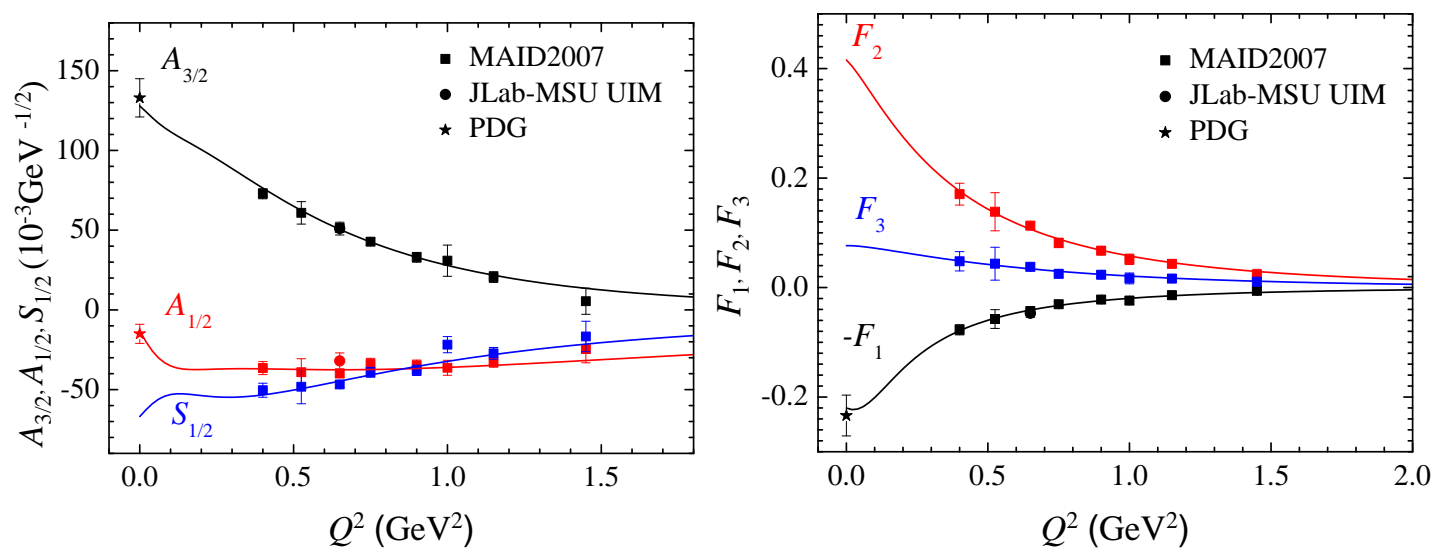

Figure 3: Helicity amplitudes and point and gauge invariant FFs for the transition $p \gamma^{*} \rightarrow N_{+}^{*}(1680)$ $\left(\chi^{2} / D O F=0.87\right)$
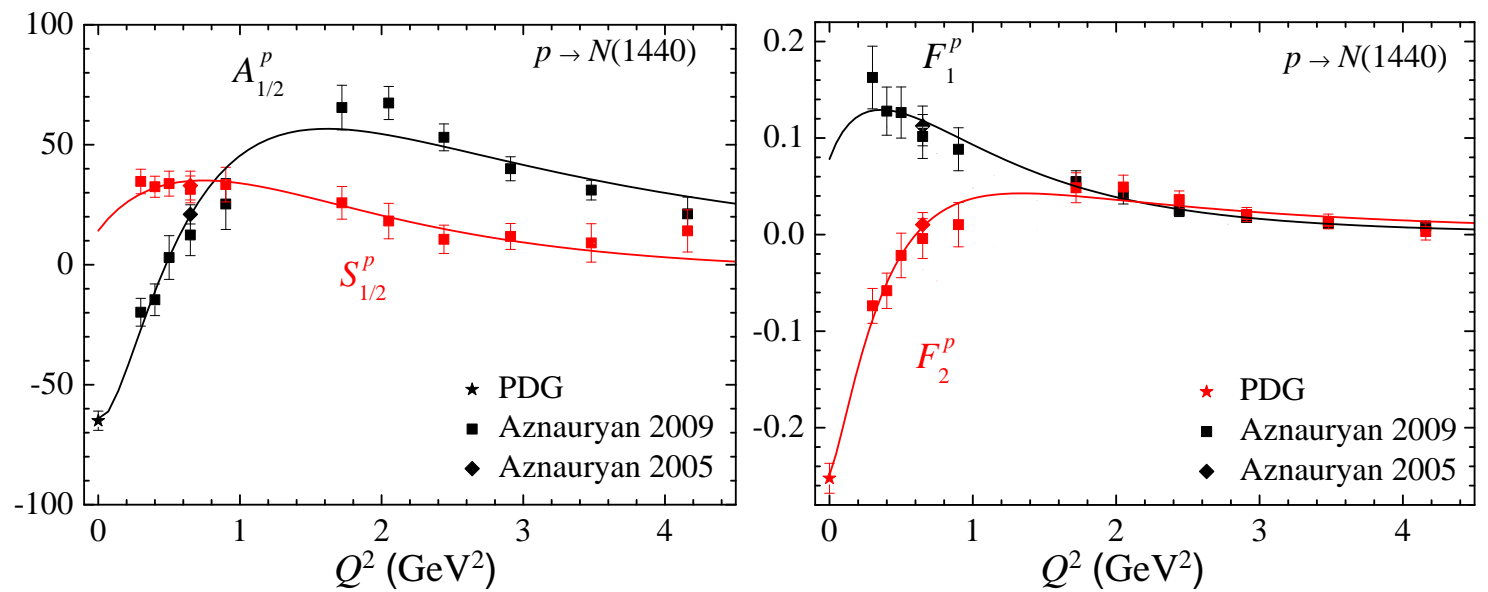

Figure 4: Helicity amplitudes and FFs for the transition $p \gamma^{*} \rightarrow N_{+}^{*}(1440)\left(\chi^{2} / D O F=0.97\right)$
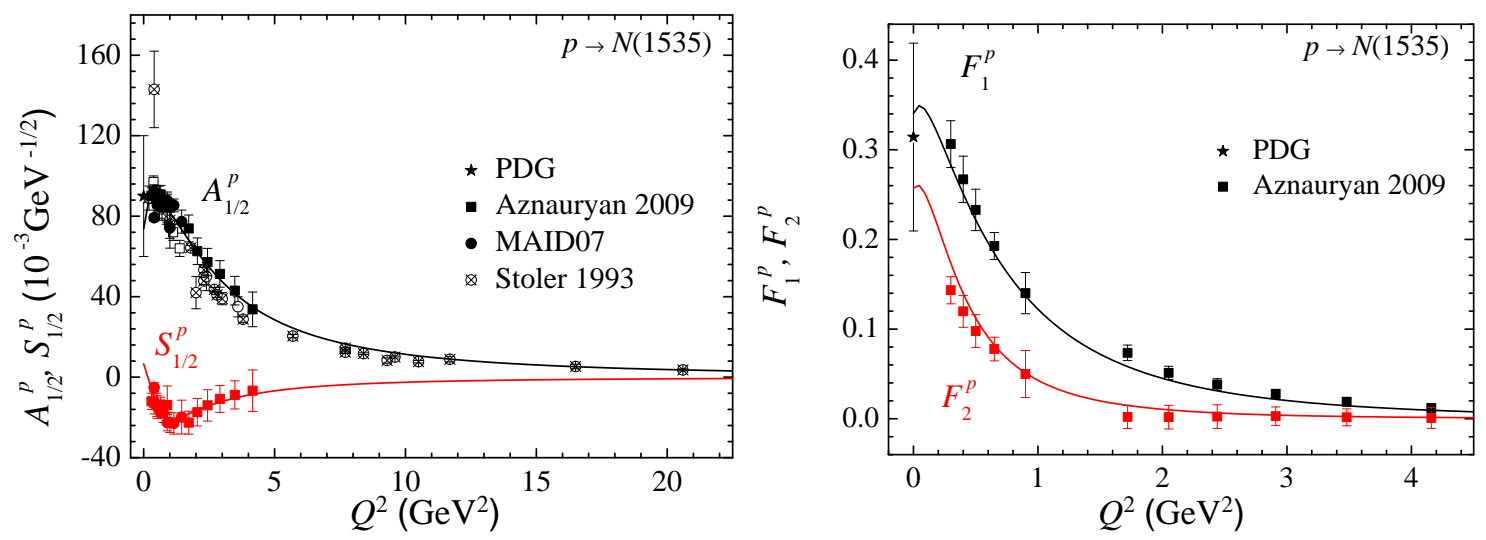

Figure 5: Helicity amplitudes and FFs for the transition $p \gamma^{*} \rightarrow N_{+}^{*}(1535)\left(\chi^{2} / D O F=0.65\right)$ 
The $Q^{2}$-dependencies of the FFs are described in the VMD model of Ref. [11]. The model agrees with the experimental data well, which supports the notion that vector-meson dominance model is applicable to the global in $Q^{2}$ description of all the nucleon electromagnetic FFs and that the higher-spin intrinsic symmetries can be used as a tool to constrain the structure of the baryonmeson vertex.

\section{References}

[1] T. Pilling, New symmetry current for massive spin-3/2 fields, Mod. Phys. Lett. A 19 (2004) 1781 [hep-ph/0404089].

[2] G. Velo, D. Zwanziger, Propagation and Quantization of Rarita-Schwinger Waves in an External Electromagnetic Potential, Phys. Rev. 186 (1969) 1337.

[3] K. Johnson, E. C. G. Sudarshan, Inconsistency of the local field theory of charged spin 3/2 particles, Ann. Phys. (N.Y.) 13 (1961) 126.

[4] L. P. S. Singh, Noncausal Propagation of Classical Rarita-Schwinger Waves, Phys. Rev. D 7, 1256 (1973).

[5] V. Pascalutsa, R. Timmermans, Field theory of nucleon to higher-spin baryon transitions, Phys. Rev. C 60 (1999) 042201(R) [nucl-th/9905065v2].

[6] G. Vereshkov, N. Volchanskiy, Low- $Q^{2}$ scaling behavior of the form-factor ratios for the $N \Delta(1232)$-transition, Phys. Lett. B 688 (2010) 168 [arXiv: 0901.0184 ].

[7] G. Vereshkov, N. Volchanskiy, Symmetries of higher-spin fields and the electromagnetic $N-N^{*}(1680)$ form factors, Phys. Rev. C 82 (2010) 045204 [arXiv: 1006.3929 ].

[8] C. E. Carlson, Electromagnetic N $\Delta$ transition at high $Q^{2}$, Phys. Rev. D 34 (1986) 2704.

[9] C. E. Carlson, J. L. Poor, Distribution amplitudes and electroproduction of the delta and other low-lying resonances, Phys. Rev. D 38 (1988) 2758.

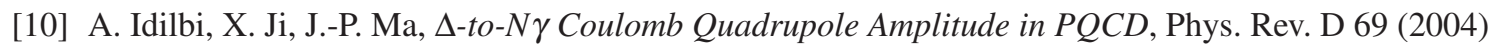
014006 [hep-ph/0308018].

[11] G. Vereshkov, N. Volchanskiy, $Q^{2}$-evolution of nucleon-to-resonance transition form factors in a QCD-inspired vector-meson-dominance model, Phys. Rev. D 76 (2007) 073007 [arXiv:0707.0051]. 